\title{
AN ELLIPTIC MACDONALD-MORRIS CONJECTURE AND MULTIPLE MODULAR HYPERGEOMETRIC SUMS
}

\author{
J. F. VAn Diejen and V. P. Spiridonov
}

\begin{abstract}
We present an elliptic Macdonald-Morris constant term conjecture in the form of an evaluation formula for a Selberg-type multiple beta integral composed of elliptic gamma functions. By multivariate residue calculus, a summation formula recently conjectured by Warnaar for a multiple modular (or elliptic) hypergeometric series is recovered. When the imaginary part of the modular parameter tends to $+\infty$, our elliptic Macdonald-Morris conjecture follows from a Selberg-type multivariate Nassrallah-Rahman integral due to Gustafson. As a consequence we arrive at a proof for the basic hypergeometric degeneration of Warnaar's sum, which amounts to a multidimensional generalization of Jackson's very-well-poised balanced terminating ${ }_{8} \Phi_{7}$ summation formula. By exploiting its modular properties, the validity of Warnaar's sum at the elliptic level is moreover verified independently for low orders in $\log (q)$ (viz. up to order 10).
\end{abstract}

\section{Introduction}

In [FT] Frenkel and Turaev introduced the concept of elliptic or modular hypergeometric series. When the imaginary part of the elliptic modulus tends to $+\infty$, these series degenerate to Heine's basic hypergeometric series [GR]. One of the key results of [FT] consists of a modular generalization of a classical summation formula due to Jackson for the terminating very-well-poised balanced ${ }_{8} \Phi_{7}$ series [GR]. Recently, Warnaar conjectured a multidimensional generalization of the Frenkel-Turaev sum [Wa]. This conjecture fits in rich line of research on multiple basic hypergeometric series, see e.g. [M1, M2, ML, A, I, Ma1, D, Sc, BF] and references therein.

In this paper we present a conjectural evaluation formula for an elliptic Selberg-type beta integral whose integrand is built of products of Ruijsenaars' elliptic gamma functions [Ru]. The integral in question may be seen as an elliptic version of a Macdonald-Morris constant term identity connected with the classical root systems $[\mathrm{Ma} 2, \mathrm{~K}]$. In the one-variable case this integral was previously introduced by one of us in [S], together with a proof valid for discrete parameter sequences. At this one-variable level the integral may be seen as an elliptic extension of a generalized Askey-Wilson integral [AW, GR] due to Nassrallah

Received May 22, 2000.

Work supported in part by the Fondo Nacional de Desarrollo Científico y Tecnológico (FONDECYT) Grants \# 1980832 and \# 7980041, the Cátedra Presidencial in Number Theory, and by the Russian Foundation for Basic Research (RFBR) Grant \# 01-00299. 
and Rahman [NR]. We show, by multivariate residue calculus in the spirit of [St, HO, BS], that Warnaar's multiple modular hypergeometric Frenkel-Turaev sum follows from our elliptic Macdonald-Morris conjecture.

When the imaginary part of the elliptic modulus tends to $+\infty$, the elliptic Selberg integral degenerates to a Selberg-type multivariate Nassrallah-Rahman integral evaluated by Gustafson [G2]. Hence, this provides us with a complete proof of the basic hypergeometric degeneration of the multiple Frenkel-Turaev sum, which amounts to a multidimensional generalization of Jackson's terminating very-well-poised balanced ${ }_{8} \Phi_{7}$ sum. This multiple basic hypergeometric sum constitutes the generalization to the ${ }_{8} \Phi_{7}$ level of a multidimensional very-wellpoised terminating ${ }_{6} \Phi_{5}$ summation formula related to summation identities of Aomoto, Ito and Macdonald [A, I, Ma1] that was presented in [D]. It is different from the multiple ${ }_{8} \Phi_{7}$ summation formulas that can be found in the works of Milne [M1, M2, ML] and Schlosser [Sc]. By invoking results from the theory of modular forms, we are moreover able to verify the validity of the multiple Frenkel-Turaev sum at the elliptic level for low orders in $\log (q)$ (up to order 10).

It is well-known that the Jackson's ${ }_{8} \Phi_{7}$ sum has important applications in the settling of normalization questions for basic hypergeometric biorthogonal rational functions [W1, W2, R1, R2]. Recently it was demonstrated that the FrenkelTuraev sum admits a similar application in the study of new types of modular hypergeometric biorthogonal rational functions on elliptic grids [SZ]. An important incentive for the present work is the search for multivariate biorthogonal rational functions that incorporate both the classical one-variable biorthogonal functions and the celebrated Macdonald polynomials [Ma2].

The plan of the paper is as follows. In Section 2 some properties of the elliptic gamma functions are recalled. The elliptic Selberg integration formula encoding our Macdonald-Morris conjecture is formulated in Section 3. In Section 4 a residue formula for the elliptic Selberg integral is presented. With the aid of the residue formula we recover the multiple modular hypergeometric Frenkel-Turaev summation formula in Section 5. We conclude with Section 6, where the modular properties and low-order behavior in $\log (q)$ of the multiple sum in question are studied.

\section{The elliptic gamma function}

In this section some elementary properties of Ruijsenaars' elliptic gamma function are recapitulated. For further information we refer to $[\mathrm{Ru}]$ and $[\mathrm{FV}]$.

Let

$$
p=e^{2 \pi i \tau}, \quad q=e^{2 \pi i \sigma},
$$

with $\operatorname{Im}(\tau), \operatorname{Im}(\sigma)>0$ (such that $0<|p|,|q|<1$ ). We introduce the double product

$$
(a ; p, q)_{\infty} \equiv \prod_{j, k=0}^{\infty}\left(1-a p^{j} q^{k}\right)
$$


For $p=0$ this double product reduces to the usual $q$-shifted factorial $(a ; q)_{\infty} \equiv$ $\prod_{k=0}^{\infty}\left(1-a q^{k}\right)[\mathrm{GR}]$. The elliptic gamma function is now defined as

$$
\Gamma(z ; p, q)=\frac{\left(p q z^{-1} ; p, q\right)_{\infty}}{(z ; p, q)_{\infty}}
$$

It satisfies the first-order difference equations

$$
\Gamma(q z ; p, q)=\theta(z ; p) \Gamma(z ; p, q), \quad \Gamma(p z ; p, q)=\theta(z ; q) \Gamma(z ; p, q)
$$

and the reflection equation

$$
\Gamma(z ; p, q) \Gamma\left(z^{-1} ; p, q\right)=\frac{1}{\theta(z ; p) \theta\left(z^{-1} ; q\right)},
$$

where the $\theta$-function is defined as

$$
\theta(z ; p)=\left(z, p z^{-1} ; p\right)_{\infty}
$$

$\left(\equiv(z ; p)_{\infty}\left(p z^{-1} ; p\right)_{\infty}\right)$. This theta function satisfies the functional relations

$$
\theta(p z ; p)=\theta\left(z^{-1} ; p\right)=-z^{-1} \theta(z ; p),
$$

and is related to the Jacobi $\theta_{1}$-function [WW] via the Jacobi triple product identity

$$
\begin{aligned}
\theta_{1}(x \mid \tau) & =2 \sum_{m=0}^{\infty}(-1)^{m} p^{(2 m+1)^{2} / 8} \sin \pi(2 m+1) x \\
& =p^{1 / 8} i e^{-\pi i x}(p ; p)_{\infty} \theta\left(e^{2 \pi i x} ; p\right)
\end{aligned}
$$

Quotients of elliptic gamma functions give rise to elliptic Pochhammer symbols defined by

$$
\theta(z ; p ; q)_{m}=\frac{\Gamma\left(z q^{m} ; p, q\right)}{\Gamma(z ; p, q)}=\prod_{j=0}^{m-1} \theta\left(z q^{j} ; p\right), \quad m \in \mathbb{N} .
$$

For $p=0$ the elliptic gamma function and elliptic Pochhammer symbol reduce to the $q$-shifted factorials $\Gamma(z ; 0, q)=1 /(z ; q)_{\infty}=1 / \prod_{j=0}^{\infty}\left(1-z q^{j}\right)$ and $\theta(z ; 0, q)_{m}=(z ; q)_{m}=\prod_{j=0}^{m-1}\left(1-z q^{j}\right)$, respectively. Following the standard short-hand conventions of basic hypergeometric analysis for the products of $q$ shifted factorials $\left(a_{1}, \ldots, a_{l} ; q\right)_{m}=\prod_{r=1}^{l}\left(a_{r} ; q\right)_{m}$, we will often make use of the compact notations:

$$
\begin{aligned}
\Gamma\left(a_{1}, \ldots, a_{l} ; p, q\right) & =\prod_{r=1}^{l} \Gamma\left(a_{r} ; p, q\right), \\
\theta\left(a_{1}, \ldots, a_{l} ; p ; q\right)_{m} & =\prod_{r=1}^{l} \theta\left(a_{r} ; p ; q\right)_{m}, \\
\theta\left(a_{1}, \ldots, a_{l} ; p\right) & =\prod_{r=1}^{l} \theta\left(a_{r} ; p\right) .
\end{aligned}
$$




\section{An elliptic Macdonald-Morris conjecture}

Let

$$
\begin{aligned}
\Delta_{n}(\mathbf{z} ; p, q)= & \frac{1}{(2 \pi i)^{n}} \prod_{1 \leq j<k \leq n} \frac{\Gamma\left(t z_{j} z_{k}, t z_{j} z_{k}^{-1}, t z_{j}^{-1} z_{k}, t z_{j}^{-1} z_{k}^{-1} ; p, q\right)}{\Gamma\left(z_{j} z_{k}, z_{j} z_{k}^{-1}, z_{j}^{-1} z_{k}, z_{j}^{-1} z_{k}^{-1} ; p, q\right)} \\
& \times \prod_{j=1}^{n} \frac{\prod_{r=0}^{4} \Gamma\left(t_{r} z_{j}, t_{r} z_{j}^{-1} ; p, q\right)}{\Gamma\left(z_{j}^{2}, z_{j}^{-2}, z_{j} t^{2 n-2} \prod_{s=0}^{4} t_{s}, z_{j}^{-1} t^{2 n-2} \prod_{s=0}^{4} t_{s} ; p, q\right)} .
\end{aligned}
$$

We conjecture that the following elliptic Selberg-type integration formula for the function $\Delta_{n}(\mathbf{z} ; p, q)$ holds true.

Conjecture (Elliptic Macdonald-Morris Identity). Let $|p|,|q|,|t|$ and $\left|t_{r}\right|$ (where $r=0, \ldots, 4$ ) be smaller than 1 with $|p q|<\left|t^{2 n-2} \prod_{s=0}^{4} t_{s}\right|$. Then,

$$
\begin{aligned}
& \int_{T^{n}} \Delta_{n}(\mathbf{z} ; p, q) \frac{d z_{1}}{z_{1}} \cdots \frac{d z_{n}}{z_{n}}= \\
& \frac{2^{n} n !}{(q ; q)_{\infty}^{n}(p ; p)_{\infty}^{n}} \prod_{j=1}^{n} \frac{\Gamma\left(t^{j} ; p, q\right)}{\Gamma(t ; p, q)} \frac{\prod_{0 \leq r<s \leq 4} \Gamma\left(t^{j-1} t_{r} t_{s} ; p, q\right)}{\prod_{r=0}^{4} \Gamma\left(t^{n+j-2} t_{r}^{-1} \prod_{s=0}^{4} t_{s} ; p, q\right)},
\end{aligned}
$$

where $T$ denotes the unit circle with positive orientation.

For $p=0$ the integrand $\Delta_{n}(\mathbf{z} ; p, q)(3.1)$ degenerates to

$$
\begin{aligned}
\Delta_{n}(\mathbf{z} ; q)= & \frac{1}{(2 \pi i)^{n}} \prod_{1 \leq j<k \leq n} \frac{\left(z_{j} z_{k}, z_{j} z_{k}^{-1}, z_{j}^{-1} z_{k}, z_{j}^{-1} z_{k}^{-1} ; q\right)_{\infty}}{\left(t z_{j} z_{k}, t z_{j} z_{k}^{-1}, t z_{j}^{-1} z_{k}, t z_{j}^{-1} z_{k}^{-1} ; q\right)_{\infty}} \\
& \times \prod_{1 \leq j \leq n} \frac{\left(z_{j}^{2}, z_{j}^{-2}, z_{j} t^{2 n-2} \prod_{s=0}^{4} t_{s}, z_{j}^{-1} t^{2 n-2} \prod_{s=0}^{4} t_{s} ; q\right)_{\infty}}{\prod_{r=0}^{4}\left(t_{r} z_{j}, t_{r} z_{j}^{-1} ; q\right)_{\infty}} .
\end{aligned}
$$

The integration formula of the conjecture then reduces to a Selberg-type multivariate Nassrallah-Rahman integral due to Gustafson [G2]

$$
\begin{aligned}
& \int_{T^{n}} \Delta_{n}(\mathbf{z} ; q) \frac{d z_{1}}{z_{1}} \cdots \frac{d z_{n}}{z_{n}}= \\
& 2^{n} n ! \prod_{j=1}^{n} \frac{(t ; q)_{\infty} \prod_{r=0}^{4}\left(t^{n+j-2} t_{r}^{-1} \prod_{s=0}^{4} t_{s} ; q\right)_{\infty}}{\left(q, t^{j} ; q\right)_{\infty} \prod_{0 \leq r<s \leq 4}\left(t_{r} t_{s} t^{j-1} ; q\right)_{\infty}}
\end{aligned}
$$

with $|q|,|t|$ and $\left|t_{r}\right|<1$ for $r=0, \ldots, 4$.

The integration conjecture claims that the constant term of the Laurent expansion of $\Delta_{n}(\mathbf{z} ; p, q)(3.1)$ in the variable $\mathbf{z}$ has the value given by the r.h.s. of (3.2). For $p=0$ the corresponding expression for the constant term of $\Delta_{n}(\mathbf{z} ; q)$ (3.3), given by the r.h.s. of Gustafson's integral (3.4), reduces for special values of the parameters $t_{r}$ to certain ( $B C$-type) constant term identities that were originally conjectured by Macdonald and Morris [Ma2, K]. This explains the name elliptic Macdonald-Morris conjecture for the above conjectural Selbergtype integration formula. 
Remark 1. For $n=1$ the integration formula of the elliptic Macdonald-Morris conjecture simplifies as

$$
\begin{aligned}
\frac{1}{2 \pi i} \int_{T} \frac{\prod_{r=0}^{4} \Gamma\left(z t_{r}, z^{-1} t_{r} ; p, q\right)}{\Gamma\left(z^{2}, z^{-2}, z \prod_{r=0}^{4} t_{r}, z^{-1} \prod_{r=0}^{4} t_{r} ; p, q\right)} \frac{d z}{z}= \\
\frac{2 \prod_{0 \leq r<s \leq 4} \Gamma\left(t_{r} t_{s} ; p, q\right)}{(q ; q)_{\infty}(p ; p)_{\infty} \prod_{r=0}^{4} \Gamma\left(t_{r}^{-1} \prod_{s=0}^{4} t_{s} ; p, q\right)}
\end{aligned}
$$

This formula was previously introduced in [S] with a proof for discrete parameter sequences. The $p=0$ degeneration of the $n=1$ integration formula amounts to a generalization of the Askey-Wilson integral [AW, GR] due to Nassrallah and Rahman $[\mathrm{NR}]$

$$
\begin{aligned}
\frac{1}{2 \pi i} \int_{T} \frac{\left(z^{2}, z^{-2}, z \prod_{r=0}^{4} t_{r}, z^{-1} \prod_{r=0}^{4} t_{r} ; q\right)_{\infty}}{\prod_{r=0}^{4}\left(t_{r} z, t_{r} z^{-1} ; q\right)_{\infty}} \frac{d z}{z}= & \\
& \frac{2 \prod_{r=0}^{4}\left(t_{r}^{-1} \prod_{s=0}^{4} t_{s} ; q\right)_{\infty}}{(q ; q)_{\infty} \prod_{0 \leq r<s \leq 4}\left(t_{r} t_{s} ; q\right)_{\infty}}
\end{aligned}
$$

\section{A residue formula}

For the parameter regime indicated in the elliptic Macdonald-Morris conjecture, the integrand $\Delta_{n}(\mathbf{z} ; p, q)(3.1)$ has poles in $z_{j}$ inside the unit circle at $\left\{t_{r} p^{l} q^{m}\right\}_{l, m \in \mathbb{N}} \quad(r=0, \ldots, 4), \quad\left\{z_{k}^{ \pm 1} t p^{l} q^{m}\right\}_{l, m \in \mathbb{N}}, \quad\left\{z_{k}^{ \pm 1} p^{l+1} q^{m+1}\right\}_{l, m \in \mathbb{N}}$, $\left\{ \pm p^{(l+1) / 2} q^{(m+1) / 2}\right\}_{l, m \in \mathbb{N}}$, and $\left\{p^{l+1} q^{m+1} t^{2-2 n} \prod_{s=0}^{4} t_{s}^{-1}\right\}_{l, m \in \mathbb{N}}$. Furthermore, due to the $z_{j} \rightarrow z_{j}^{-1}$ reflection-invariance of the integrand, the poles located outside the unit circle are related to these by inversion. We will now dilate the parameter $t_{0}$ from the regime $\left|t_{0}\right|<1$ to the regime $\left|t_{0}\right|>1$. In the process (a finite number of) poles will move from the interior to the exterior of the unit circle and vice versa. Specifically, if $q^{-N}<\left|t_{0}\right|<q^{-N-1}$ for some integer $N \in \mathbb{N}$ and $0<q<1$. Then, for $p$ sufficiently small (viz. $p<1 /\left|t_{0}\right|$ ), we see that the poles in $z_{j}$ at $t_{0} p^{l} q^{m}$ relocate to the exterior of the unit circle iff $l=0$ and $m=0, \ldots, N$. Correspondingly, the poles related to these by inversion have moved from the exterior to the interior of the unit circle. The following theorem provides a residue formula that keeps track of such pole movements across the integration contour. Analogous residue formulas for Selberg-type multivariate integration measures can be found in the works [St, HO, BS].

Theorem 1 (Residue Formula). Let $\Delta_{n}(\mathbf{z} ; p, q)$ be given by (3.1) with $0<q$, $t<$ 1 and $t_{0}, \ldots, t_{4}$ generic such that $\#\left\{\arg \left(t_{r}\right), \arg \left(t_{r}^{-1}\right) \mid r=0, \ldots, 4\right\}=10$ and $t_{r}^{-1} \prod_{s=0}^{4} t_{s} \notin\left[1,+\infty\left[\right.\right.$ for $r=0, \ldots, 4$. Let us furthermore pick $\left|t_{0}\right|>1$ and $\left|t_{r}\right|<1$ for $r=1, \ldots, 4$, and let us assume that $p$ is sufficiently small such that 
$0<p<\min \left(\left|t_{0}\right|^{-1}, q^{-1} t^{2 n-2} \prod_{s=0}^{4}\left|t_{s}\right|\right)$. Then we have that

$$
\begin{aligned}
\int_{C^{n}} \Delta_{n}(\mathbf{z} ; p, q) \frac{d z_{1}}{z_{1}} \cdots \frac{d z_{n}}{z_{n}}= \\
\sum_{m=0}^{n} 2^{m} m !\left(\begin{array}{c}
n \\
m
\end{array}\right) \sum_{\substack{0 \leq \lambda_{1} \leq \cdots \leq \lambda_{m} \\
\left|\tau_{m} q^{\lambda_{m}}\right|>1}} \int_{T^{n-m}} \mu_{m}(\lambda, \mathbf{z} ; p ; q) \frac{d z_{1}}{z_{1}} \cdots \frac{d z_{n-m}}{z_{n-m}},
\end{aligned}
$$

where $\tau_{j}=t_{0} t^{j-1}, j=1, \ldots, n$,

$$
\mu_{m}(\lambda, \mathbf{z} ; p ; q)=\kappa_{m} \nu_{m}(\lambda ; p ; q) \delta_{m, n-m}(\lambda, \mathbf{z}) \Delta_{n-m}(\mathbf{z} ; p, q),
$$

with

$$
\begin{aligned}
& \kappa_{m}=\prod_{1 \leq j<k \leq m} \frac{\Gamma\left(t \tau_{k} \tau_{j}^{-1}, t \tau_{k}^{-1} \tau_{j}^{-1} ; p, q\right)}{\Gamma\left(\tau_{k} \tau_{j}^{-1}, \tau_{k}^{-1} \tau_{j}^{-1} ; p, q\right)} \\
& \times \prod_{j=1}^{m} \frac{\prod_{r=1}^{4} \Gamma\left(t_{r} \tau_{j}, t_{r} \tau_{j}^{-1} ; p, q\right)}{(q ; q)_{\infty}(p ; p)_{\infty} \Gamma\left(\tau_{j}^{-2}, \tau_{j}^{-1} t^{2 n-2} \prod_{s=0}^{4} t_{s}, \tau_{j} t^{2 n-2} \prod_{s=0}^{4} t_{s} ; p, q\right)}, \\
& \nu_{m}(\lambda ; p ; q)=q^{\sum_{j=1}^{m} \lambda_{j}} t^{2 \sum_{j=1}^{m}(n-j) \lambda_{j}} \prod_{1 \leq j<k \leq m}\left(\frac{\theta\left(\tau_{k} \tau_{j} q^{\lambda_{k}+\lambda_{j}}, \tau_{k} \tau_{j}^{-1} q^{\lambda_{k}-\lambda_{j}} ; p\right)}{\theta\left(\tau_{k} \tau_{j}, \tau_{k} \tau_{j}^{-1} ; p\right)}\right. \\
& \left.\times \frac{\theta\left(t \tau_{k} \tau_{j} ; p ; q\right)_{\lambda_{k}+\lambda_{j}}}{\theta\left(q t^{-1} \tau_{k} \tau_{j} ; p ; q\right)_{\lambda_{k}+\lambda_{j}}} \frac{\theta\left(t \tau_{k} \tau_{j}^{-1} ; p ; q\right)_{\lambda_{k}-\lambda_{j}}}{\theta\left(q t^{-1} \tau_{k} \tau_{j}^{-1} ; p ; q\right)_{\lambda_{k}-\lambda_{j}}}\right) \\
& \times \prod_{j=1}^{m}\left(\frac{\theta\left(\tau_{j}^{2} q^{2 \lambda_{j}} ; p\right)}{\theta\left(\tau_{j}^{2} ; p\right)} \prod_{r=0}^{5} \frac{\theta\left(t_{r} \tau_{j} ; p ; q\right)_{\lambda_{j}}}{\theta\left(q t_{r}^{-1} \tau_{j} ; p ; q\right)_{\lambda_{j}}}\right)
\end{aligned}
$$

and

$$
\delta_{m, n-m}(\lambda, \mathbf{z})=\prod_{\substack{1 \leq j \leq m \\ 1 \leq k \leq n-m}} \frac{\Gamma\left(t \tau_{j} q^{\lambda_{j}} z_{k}, t \tau_{j} q^{\lambda_{j}} z_{k}^{-1}, t \tau_{j}^{-1} q^{-\lambda_{j}} z_{k}, t \tau_{j}^{-1} q^{-\lambda_{j}} z_{k}^{-1} ; p, q\right)}{\Gamma\left(\tau_{j} q^{\lambda_{j}} z_{k}, \tau_{j} q^{\lambda_{j}} z_{k}^{-1}, \tau_{j}^{-1} q^{-\lambda_{j}} z_{k}, \tau_{j}^{-1} q^{-\lambda_{j}} z_{k}^{-1} ; p, q\right)} .
$$

Here $T$ represents the positively oriented unit circle and the integration contour $C \subset \mathbb{C}$ is a (smooth) positively oriented Jordan curve around zero such that (i) every half-line parting from zero intersects $C$ just once, (ii) $C^{-1}:=\{z \in$ $\left.\mathbb{C} \mid z^{-1} \in C\right\}=C$, and (iii) $C$ separates the poles in $z_{j}$ at $\left\{t_{r} p^{l} q^{m}\right\}_{l, m \in \mathbb{N}}$ $(r=0, \ldots, 4)$, (all in the interior of $C$ ) from those related to it by inversion (all in the exterior of $C$ ). Furthermore, $t_{5}\left(\right.$ in $\nu_{m}(\lambda ; p ; q)$ ) is determined by $q, t$ and $t_{0}, \ldots, t_{4}$ via the balancing condition $q^{-1} t^{2 n-2} \prod_{r=0}^{5} t_{r}=1$. (Notice that the conditions on the parameters and the integration contour guarantee that $C$ also separates the remaining poles in $z_{j}$ at $\left\{z_{k}^{ \pm 1} t p^{l} q^{m}\right\}_{l, m \in \mathbb{N}},\left\{z_{k}^{ \pm 1} p^{l+1} q^{m+1}\right\}_{l, m \in \mathbb{N}}$, $\left\{ \pm p^{(l+1) / 2} q^{(m+1) / 2}\right\}_{l, m \in \mathbb{N}}, \quad$ and $\quad\left\{p^{l+1} q^{m+1} t^{2-2 n} \prod_{s=0}^{4} t_{s}^{-1}\right\}_{l, m \in \mathbb{N}}$ (in the interior) from the ones related to these by inversion (in the exterior).) 
Proof. The proof of the theorem hinges on a residue formula for multivariate Askey-Wilson-type integrals due to Stokman [St]. For the precise details we refer to the appendix below.

Theorem 1 describes how one picks up residues in the elliptic Selberg integral when the cycle is deformed from $C$ to $T$. The genericity conditions on the parameters serve to ensure that the poles at issue are simple. The combinatorial factor $2^{m} m$ ! $\left(\begin{array}{c}n \\ m\end{array}\right)$ appearing in the residue formula is a consequence of the $S_{n} \ltimes \mathbb{Z}_{2}^{n}$ Weyl-group symmetry of the integrand. (Here the group $S_{n}$ acts on the variables $z_{1}, \ldots, z_{n}$ by permutation and the $\mathbb{Z}_{2}$ action corresponds to the inversion $z_{j} \rightarrow$ $z_{j}^{-1}$.) This combinatorial factor decomposes as $\left(\begin{array}{l}n \\ m\end{array}\right)$ (the number of ways to select $m$ out of $n$ cycles contributing to the residue factor), $m$ ! (the number of ways to order the $m$ integration variables of the cycles from which residues are picked up) and $2^{m}$ (originating from the $z_{j} \rightarrow z_{j}^{-1}$ reflection-invariance, which implies that each time a residue is picked up the cycle actually moves over a pair of poles with opposite residue: one entering and one leaving the interior of the contour).

Remark 2. It is straightforward to extend the elliptic Macdonald-Morris conjecture of the previous section to parameter domains and integration cycles of the type in Theorem 1 by analyticity. Indeed, assuming the genericity condition on the arguments of the parameters $t_{r}^{ \pm 1}$ and $0<q, t<1$ with $p$ sufficiently small, we may dilate the $t_{r}$ radially from the interior of the unit circle to the exterior while at the same time deforming the integration contour $C$ starting from the unit circle such that the conditions (i)-(iii) of the theorem remain fulfilled (so as to avoid crossing over poles). By analyticity in the parameters, the evaluation formula for our elliptic Selberg-type integral will then hold for the modified integration contours and parameter values provided it holds for the unit circle and the parameter domains specified in the conjecture of Section 3.

Remark 3. For $n=1$ the residue formula of Theorem 1 simplifies to

$$
\begin{aligned}
& \frac{1}{2 \pi i} \int_{C} \frac{\prod_{r=0}^{4} \Gamma\left(z t_{r}, z^{-1} t_{r} ; p, q\right)}{\Gamma\left(z^{2}, z^{-2}, z \prod_{r=0}^{4} t_{r}, z^{-1} \prod_{r=0}^{4} t_{r} ; p, q\right)} \frac{d z}{z}= \\
& \frac{1}{2 \pi i} \int_{T} \frac{\prod_{r=0}^{4} \Gamma\left(z t_{r}, z^{-1} t_{r} ; p, q\right)}{\Gamma\left(z^{2}, z^{-2}, z \prod_{r=0}^{4} t_{r}, z^{-1} \prod_{r=0}^{4} t_{r} ; p, q\right)} \frac{d z}{z}+2 \kappa \sum_{\substack{\lambda \geq 0 \\
\left|t_{0} q^{\lambda}\right|>1}} \nu(\lambda ; p ; q),
\end{aligned}
$$

where

$$
\begin{aligned}
\kappa & =\frac{\prod_{r=1}^{4} \Gamma\left(t_{r} t_{0}, t_{r} t_{0}^{-1} ; p, q\right)}{(q ; q)_{\infty}(p ; p)_{\infty} \Gamma\left(t_{0}^{-2}, t_{0}^{-1} \prod_{r=0}^{4} t_{r}, t_{0} \prod_{r=0}^{4} t_{r} ; p, q\right)}, \\
\nu(\lambda ; p ; q) & =q^{\lambda} \frac{\theta\left(t_{0}^{2} q^{2 \lambda} ; p\right)}{\theta\left(t_{0}^{2} ; p\right)} \prod_{r=0}^{5} \frac{\theta\left(t_{r} t_{0} ; p ; q\right)_{\lambda}}{\theta\left(q t_{r}^{-1} t_{0} ; p ; q\right)_{\lambda}},
\end{aligned}
$$


and $q^{-1} \prod_{r=0}^{5} t_{r}=1$. This is the elliptic generalization of known residue formulas for the Askey-Wilson and Nassrallah-Rahman integrals [AW, R2].

\section{Multiple modular hypergeometric sums}

By combining the elliptic Macdonald-Morris conjecture of Section 3 with the residue formula of Section 4, we arrive at the following multidimensional modular hypergeometric Frenkel-Turaev sum.

Corollary 2 (Multiple Frenkel-Turaev Sum). Let $N \in \mathbb{N}$. For parameters subject to

$$
\begin{aligned}
q^{-1} t^{2 n-2} \prod_{r=0}^{5} t_{r}=1 & & \text { (balancing condition }) \\
q^{N} t^{n-1} t_{0} t_{4}=1 & & \text { (truncation condition })
\end{aligned}
$$

one has that

$$
\sum_{0 \leq \lambda_{1} \leq \lambda_{2} \leq \cdots \leq \lambda_{n} \leq N} \nu_{n}(\lambda ; p ; q)=\mathcal{N}_{n}(p ; q)
$$

(as a meromorphic identity in the parameters subject to the relation (5.1)), where $\nu_{n}(\lambda ; p ; q)$ is as stated in Theorem 1 and

$$
\begin{aligned}
\mathcal{N}_{n}(p ; q)= & \prod_{1 \leq j<k \leq n} \frac{\theta\left(q \tau_{k} \tau_{j}, q t \hat{\tau}_{k}^{-1} \hat{\tau}_{j}^{-1} ; p ; q\right)_{N}}{\theta\left(q t^{-1} \tau_{k} \tau_{j}, q \hat{\tau}_{k}^{-1} \hat{\tau}_{j}^{-1} ; p ; q\right)_{N}} \\
& \times \prod_{1 \leq j \leq n} \frac{\theta\left(q \tau_{j}^{2} ; p ; q\right)_{N} \prod_{r=1}^{3} \theta\left(q \hat{t}_{r} \hat{\tau}_{j}^{-1} ; p ; q\right)_{N}}{\theta\left(q \hat{\tau}_{j}^{-2} ; p ; q\right)_{N} \prod_{r=1}^{3} \theta\left(q t_{r}^{-1} \tau_{j} ; p ; q\right)_{N}} \\
= & \prod_{j=1}^{n} \frac{\theta\left(q t^{n+j-2} t_{0}^{2} ; p ; q\right)_{N} \prod_{1 \leq r<s \leq 3} \theta\left(q t^{1-j} t_{r}^{-1} t_{s}^{-1} ; p ; q\right)_{N}}{\theta\left(q t^{2-n-j} \prod_{r=0}^{3} t_{r}^{-1} ; p ; q\right)_{N} \prod_{r=1}^{3} \theta\left(q t^{j-1} t_{0} t_{r}^{-1} ; p ; q\right)_{N}} .
\end{aligned}
$$

Here we have employed the dual quantities $\hat{t}_{0}=\left(t_{0} t_{1} t_{2} t_{3}\right)^{1 / 2}, \hat{t}_{1}=\left(t_{0} t_{1} t_{2}^{-1} t_{3}^{-1}\right)^{1 / 2}$, $\hat{t}_{2}=\left(t_{0} t_{1}^{-1} t_{2} t_{3}^{-1}\right)^{1 / 2}, \hat{t}_{3}=\left(t_{0} t_{1}^{-1} t_{2}^{-1} t_{3}\right)^{1 / 2}$ and the notations $\tau_{j}=t_{0} t^{j-1}, \hat{\tau}_{j}=$ $\hat{t}_{0} t^{j-1}, j=1, \ldots, n$.

Proof. Let us choose the parameters in compliance with the conditions in Theorem 1 such that $t^{1-n} q^{-N}<\left|t_{0}\right|<t^{1-n} q^{-N-1}$ for some $N \in \mathbb{N}$. Division of the residue formula (4.1) by $2^{n} n ! \kappa_{n}$, and letting $t_{4}$ tend to $t_{0}^{-1} t^{1-n} q^{-N}$, then entails the stated summation formula. To see this, one first notes that the constant $\kappa_{m}$ may be rewritten as

$$
\begin{aligned}
& \kappa_{m}= \\
& \frac{1}{(q ; q)_{\infty}^{m}(p ; p)_{\infty}^{m}} \prod_{j=1}^{m} \frac{\Gamma\left(t^{j} ; p, q\right) \prod_{r=1}^{4} \Gamma\left(t^{j-1} t_{0} t_{r}, t^{1-j} t_{0}^{-1} t_{r} ; p, q\right)}{\Gamma\left(t, t^{2-m-j} t_{0}^{-2}, t^{2 n+j-3} t_{0}^{2} \prod_{r=1}^{4} t_{r}, t^{2 n-j-1} \prod_{r=1}^{4} t_{r} ; p, q\right)}
\end{aligned}
$$

(by cancelling common terms in the numerator and denominator). It is clear from this representation that the quotient $\kappa_{m} / \kappa_{n}$ with $m<n$ converges to zero as $t_{4}$ goes to $t_{0}^{-1} t^{1-n} q^{-N}$. (This is because we have a pole in $\kappa_{n}$ at 
$t_{4}=t_{0}^{-1} t^{1-n} q^{-N}$ —stemming from the factor $\Gamma\left(t^{n-1} t_{0} t_{4} ; p ; q\right)$ - that is missing in $\kappa_{m}$ when $m<n$.) Hence, since the integral

$$
\int_{T^{n-m}} \Delta_{n-m}(\mathbf{z} ; p, q) \delta_{m, n-m}(\lambda, \mathbf{z}) \frac{d z_{1}}{z_{1}} \cdots \frac{d z_{n-m}}{z_{n-m}}
$$

and the factor $\nu_{m}(\lambda ; p ; q)$ remain bounded as $t_{4} \rightarrow t_{0}^{-1} t^{1-n} q^{-N}$, we conclude that the terms originating from the r.h.s. of the residue formula tend to zero in the limit when $m<n$. The terms with $m=n$ on the other hand give rise to the sum $\sum_{0 \leq \lambda_{1} \leq \cdots \leq \lambda_{n} \leq N} \nu_{n}(\lambda ; p ; q)$, which is precisely the series on the 1.h.s. of our corollary.

To compute the corresponding limit of the l.h.s. of the residue formula, we employ the elliptic Macdonald-Morris conjecture to evaluate the integral under consideration (cf. Remark 2 above). Division by $2^{n} n ! \kappa_{n}$ then yields

$$
\prod_{j=1}^{n} \frac{\Gamma\left(t^{2-n-j} t_{0}^{-2}, t^{2 n+j-3} t_{0}^{2} \prod_{r=1}^{4} t_{r} ; p, q\right) \prod_{1 \leq r<s \leq 4} \Gamma\left(t^{j-1} t_{r} t_{s} ; p, q\right)}{\prod_{r=1}^{4} \Gamma\left(t^{1-j} t_{0}^{-1} t_{r}, t^{n+j-2} t_{r}^{-1} \prod_{s=1}^{4} t_{s} ; p, q\right)}
$$

which converges to the r.h.s. of the summation formula stated by the corollary in the limit $t_{4} \rightarrow t_{0}^{-1} t^{1-n} q^{-N}$.

In summary, this proves that the elliptic Macdonald-Morris conjecture implies the stated summation formula for parameter values with restrictions stemming from the conditions in Theorem 1. By analyticity, the result is then extended to a meromorphic identity in the complex parameters $q, t$ and $t_{r}, r=0, \ldots, 5$ subject only to the balancing and truncation conditions in (5.1).

The multiple Frenkel-Turaev sum was first conjectured by Warnaar in [Wa]. Since for $p=0$ our elliptic Macdonald-Morris conjecture reduces to Gustafson's multiple Nassrallah-Rahman integral (3.4), the above considerations provide us - via the $p=0$ version of the residue formula of Theorem 1-with a complete proof of Warnaar's conjecture in the basic hypergeometric limit $p \rightarrow 0$ :

Theorem 3 (Multiple ${ }_{8} \Phi_{7}$ Jackson Sum). Let $N \in \mathbb{N}$. For parameters subject to the balancing and truncation conditions in (5.1), one has that

$$
\sum_{0 \leq \lambda_{1} \leq \lambda_{2} \leq \cdots \leq \lambda_{n} \leq N} \nu_{n}(\lambda ; q)=\mathcal{N}_{n}(q)
$$


where

$$
\begin{aligned}
\nu_{n}(\lambda ; q)= & \\
q^{\sum_{j=1}^{n} \lambda_{j}} t^{2 \sum_{j=1}^{n}(n-j) \lambda_{j}} \prod_{1 \leq j<k \leq n} & \left(\frac{1-\tau_{k} \tau_{j} q^{\lambda_{k}+\lambda_{j}}}{1-\tau_{k} \tau_{j}} \frac{1-\tau_{k} \tau_{j}^{-1} q^{\lambda_{k}-\lambda_{j}}}{1-\tau_{k} \tau_{j}^{-1}}\right. \\
\times & \left.\frac{\left(t \tau_{k} \tau_{j} ; q\right)_{\lambda_{k}+\lambda_{j}}}{\left(q t^{-1} \tau_{k} \tau_{j} ; q\right)_{\lambda_{k}+\lambda_{j}}} \frac{\left(t \tau_{k} \tau_{j}^{-1} ; q\right)_{\lambda_{k}-\lambda_{j}}}{\left(q t^{-1} \tau_{k} \tau_{j}^{-1} ; q\right)_{\lambda_{k}-\lambda_{j}}}\right) \\
& \times \prod_{1 \leq j \leq n}\left(\frac{1-\tau_{j}^{2} q^{2 \lambda_{j}}}{1-\tau_{j}^{2}} \prod_{r=0}^{5} \frac{\left(t_{r} \tau_{j} ; q\right)_{\lambda_{j}}}{\left(q t_{r}^{-1} \tau_{j} ; q\right)_{\lambda_{j}}}\right)
\end{aligned}
$$

and

$$
\begin{aligned}
\mathcal{N}_{n}(q)= & \prod_{1 \leq j<k \leq n} \frac{\left(q \tau_{k} \tau_{j}, q t \hat{\tau}_{k}^{-1} \hat{\tau}_{j}^{-1} ; q\right)_{N}}{\left(q t^{-1} \tau_{k} \tau_{j}, q \hat{\tau}_{k}^{-1} \hat{\tau}_{j}^{-1} ; q\right)_{N}} \\
& \times \prod_{1 \leq j \leq n} \frac{\left(q \tau_{j}^{2} ; q\right)_{N} \prod_{r=1}^{3}\left(q \hat{t}_{r} \hat{\tau}_{j}^{-1} ; q\right)_{N}}{\left(q \hat{\tau}_{j}^{-2} ; q\right)_{N} \prod_{r=1}^{3}\left(q t_{r}^{-1} \tau_{j} ; q\right)_{N}} \\
= & \prod_{1 \leq j \leq n} \frac{\left(q t^{n+j-2} t_{0}^{2} ; q\right)_{N} \prod_{1 \leq r<s \leq 3}\left(q t^{1-j} t_{r}^{-1} t_{s}^{-1} ; q\right)_{N}}{\left(q t^{2-n-j} \prod_{r=0}^{3} t_{r}^{-1} ; q\right)_{N} \prod_{r=1}^{3}\left(q t^{j-1} t_{0} t_{r}^{-1} ; q\right)_{N}}
\end{aligned}
$$

(and with the dual parameters $\hat{t}_{r}, r=1, \ldots, 3$ and $\tau_{j}, \hat{\tau}_{j}, j=1, \ldots, n$ being as stated in Corollary 2).

The identity in Theorem 3 holds as a rational identity in the parameters $q, t, t_{r}(r=0, \ldots, 5)$ subject to the relations in (5.1). After elimination of $t_{4}, t_{5}$ by means of the relations (5.1), and performing the limit $t_{3} \rightarrow \infty$, the summation formula in Theorem 3 reduces to the terminating multiple ${ }_{6} \Phi_{5}$ sum of [D, Theorem 3] (which, in turn, is related to certain multidimensional summation formulas associated to root systems due to Aomoto, Ito, and Macdonald [A, I, Ma1]). Recently, it was shown by Stokman [St] that the terminating multiple ${ }_{6} \Phi_{5}$ sum of $[\mathrm{D}]$ can be obtained via residue calculus from Gustafson's multivariate Askey-Wilson integral [G1, K] by means of a method similar to the one followed in the present paper. From this perspective, our proof of Theorem 3 may be seen as a generalization of the method of [St] to the multiple ${ }_{8} \Phi_{7}$ level.

Other multidimensional generalizations of the Jackson ${ }_{8} \Phi_{7}$ sum that are different from the one in Theorem 3 can be found in the works of Milne and Schlosser $[\mathrm{M} 1, \mathrm{M} 2, \mathrm{ML}, \mathrm{Sc}]$. 
Remark 4. For $n=1$ the summations of Corollary 2 and Theorem 3 simplify as

$$
\begin{aligned}
\sum_{\lambda=0}^{N} \frac{\theta\left(t_{0}^{2} q^{2 \lambda} ; p\right)}{\theta\left(t_{0}^{2} ; p\right)} \frac{\prod_{r=0}^{5} \theta\left(t_{0} t_{r} ; p ; q\right)_{\lambda}}{\prod_{r=0}^{5} \theta\left(q t_{0} t_{r}^{-1} ; p ; q\right)_{\lambda}} q^{\lambda}= \\
\frac{\theta\left(q t_{0}^{2} ; p ; q\right)_{N} \prod_{1 \leq r<s \leq 3} \theta\left(q t_{r}^{-1} t_{s}^{-1} ; p ; q\right)_{N}}{\theta\left(q t_{0}^{-1} t_{1}^{-1} t_{2}^{-1} t_{3}^{-1} ; p ; q\right)_{N} \prod_{r=1}^{3} \theta\left(q t_{0} t_{r}^{-1} ; p ; q\right)_{N}}
\end{aligned}
$$

and

$$
\begin{aligned}
\sum_{\lambda=0}^{N}\left(\frac{1-t_{0}^{2} q^{2 \lambda}}{1-t_{0}^{2}}\right) \frac{\prod_{r=0}^{5}\left(t_{0} t_{r} ; q\right)_{\lambda}}{\prod_{r=0}^{5}\left(q t_{0} t_{r}^{-1} ; q\right)_{\lambda}} q^{\lambda} & = \\
& \frac{\left(q t_{0}^{2} ; q\right)_{N} \prod_{1 \leq r<s \leq 3}\left(q t_{r}^{-1} t_{s}^{-1} ; q\right)_{N}}{\left(q t_{0}^{-1} t_{1}^{-1} t_{2}^{-1} t_{3}^{-1} ; q\right)_{N} \prod_{r=1}^{3}\left(q t_{0} t_{r}^{-1} ; q\right)_{N}}
\end{aligned}
$$

where the parameters are assumed to satisfy the balancing condition $q^{-1} \prod_{r=0}^{5} t_{r}=1$ and the truncation condition $q^{N} t_{0} t_{4}=1$. The sum in (5.8a) amounts to the modular hypergeometric summation formula of Frenkel and Turaev $[\mathrm{FT}]$ and the sum in $(5.8 \mathrm{~b})$ boils down to the classical very-well-poised balanced terminating ${ }_{8} \Phi_{7}$ sum due to Jackson [GR].

\section{Modularity}

To simplify the comparison with the results of [FT, SZ], we rewrite the multiple Frenkel-Turaev summation of Corollary 2 in terms of elliptic numbers, which are defined as

$$
[x ; \sigma, \tau] \equiv \frac{\theta_{1}(\sigma x \mid \tau)}{\theta_{1}(\sigma \mid \tau)}=q^{(1-x) / 2} \frac{\left(q^{x}, p q^{-x} ; p\right)_{\infty}}{\left(q, p q^{-1} ; p\right)_{\infty}} .
$$

In the following we often suppress the dependence on $\sigma, \tau$ by employing the abbreviated notation $[x] \equiv[x ; \sigma, \tau]$. The corresponding elliptic analogue of the Pochhammer symbol can then be introduced as follows

$$
\begin{aligned}
{[x]_{m} } & =\prod_{j=0}^{m-1}[x+j], \quad[x]_{0}=1, \\
{\left[g_{1}, \ldots, g_{l}\right]_{m} } & =\prod_{r=1}^{l}\left[g_{r}\right]_{m} .
\end{aligned}
$$

We now define a multiple modular analogue of the very-well-poised balanced terminating basic hypergeometric ${ }_{l+1} \Phi_{l}$ series as

$$
{ }_{l+1} E_{l}^{(n)}\left(g ; g_{0} ; g_{1}, \ldots, g_{l-3} ; \sigma, \tau\right) \equiv \sum_{0 \leq \lambda_{1} \leq \lambda_{2} \leq \cdots \leq \lambda_{n} \leq N} l+1 \nu_{l}^{(n)}(\lambda ; \sigma, \tau),
$$


where

$$
\begin{aligned}
{ }_{l+1} \nu_{l}^{(n)}(\lambda ; \sigma, \tau)= & \prod_{1 \leq j<k \leq n}\left(\frac{\left[\rho_{k}+\rho_{j}+\lambda_{k}+\lambda_{j}, \rho_{k}-\rho_{j}+\lambda_{k}-\lambda_{j}\right]}{\left[\rho_{k}+\rho_{j}, \rho_{k}-\rho_{j}\right]}\right. \\
& \left.\times \frac{\left[g+\rho_{k}+\rho_{j}\right]_{\lambda_{k}+\lambda_{j}}\left[g+\rho_{k}-\rho_{j}\right]_{\lambda_{k}-\lambda_{j}}}{\left[1-g+\rho_{k}+\rho_{j}\right]_{\lambda_{k}+\lambda_{j}}\left[1-g+\rho_{k}-\rho_{j}\right]_{\lambda_{k}-\lambda_{j}}}\right) \\
& \times \prod_{j=1}^{n}\left(\frac{\left[2 \rho_{j}+2 \lambda_{j}\right]}{\left[2 \rho_{j}\right]} \prod_{r=0}^{l-2} \frac{\left[g_{r}+\rho_{j}\right]_{\lambda_{j}}}{\left[1-g_{r}+\rho_{j}\right]_{\lambda_{j}}}\right),
\end{aligned}
$$

with $\rho_{j} \equiv(j-1) g+g_{0}, j=1, \ldots, n$ and parameters $g, g_{r}$ subject to the constraints

$$
\begin{aligned}
(2 n-2) g+\sum_{r=0}^{l-2} g_{r}-(l-5) / 2=0 & & \text { (balancing condition) } \\
(n-1) g+g_{0}+g_{l-3}+N=0 & & \text { (truncation condition). }
\end{aligned}
$$

(Instead of $g_{l-3}$ one may actually use any of the parameters $g_{1}, \ldots, g_{l-3}$ in the truncation condition.) For $n=1$ this is the modular hypergeometric series of Frenkel and Turaev [FT]:

$$
{ }_{l+1} E_{l}\left(g_{0} ; g_{1}, \ldots, g_{l-3} ; \sigma, \tau\right)=\sum_{\lambda=0}^{N} \frac{\left[2 g_{0}+2 \lambda\right]}{\left[2 g_{0}\right]} \prod_{r=0}^{l-2} \frac{\left[g_{0}+g_{r}\right]_{\lambda}}{\left[1+g_{0}-g_{r}\right]_{\lambda}},
$$

where $g_{0}+\cdots+g_{l-2}-(l-5) / 2=0$ and $g_{0}+g_{l-3}+N=0$.

The summation conjecture of Corollary 2 can now be reformulated as

$$
\begin{aligned}
& \text { (6.7) }{ }_{8} E_{7}^{(n)}\left(g ; g_{0} ; g_{1}, \ldots, g_{4} ; \sigma, \tau\right) \\
& =\prod_{1 \leq j<k \leq n} \frac{\left[1+\rho_{k}+\rho_{j}, 1+g-\hat{\rho}_{k}-\hat{\rho}_{j}\right]_{N}}{\left[1-\hat{\rho}_{k}-\hat{\rho}_{j}, 1-g+\rho_{k}+\rho_{j}\right]_{N}} \prod_{j=1}^{n} \frac{\left[1+2 \rho_{j}\right]_{N} \prod_{r=1}^{3}\left[1+\hat{g}_{r}-\hat{\rho}_{j}\right]_{N}}{\left[1-2 \hat{\rho}_{j}\right]_{N} \prod_{r=1}^{3}\left[1-g_{r}+\rho_{j}\right]_{N}} \\
& =\prod_{j=1}^{n} \frac{\left[1+(n+j-2) g+2 g_{0}\right]_{N} \prod_{1 \leq r<s \leq 3}\left[1-(j-1) g-g_{r}-g_{s}\right]_{N}}{\left[1-(n+j-2) g-\sum_{r=0}^{3} g_{r}\right]_{N} \prod_{r=1}^{3}\left[1+(j-1) g+g_{0}-g_{r}\right]_{N}},
\end{aligned}
$$

where $\hat{\rho}_{j}=(j-1) g+\hat{g}_{0}(j=1, \ldots, n), \hat{g}_{0}=\left(g_{0}+g_{1}+g_{2}+g_{3}\right) / 2$, $\hat{g}_{1}=\left(g_{0}+g_{1}-g_{2}-g_{3}\right) / 2, \hat{g}_{2}=\left(g_{0}-g_{1}+g_{2}-g_{3}\right) / 2$, and $\hat{g}_{3}=\left(g_{0}-g_{1}-g_{2}+g_{3}\right) / 2$, and where the connection between the current parameters and those of Section 5 is given by $p=\exp (2 \pi i \tau), q=\exp (2 \pi i \sigma)$, and $t=q^{g}, t_{r}=q^{g_{r}}(r=0, \ldots, 5)$.

Let us abbreviate ${ }_{l+1} E_{l}^{(n)}\left(g ; g_{0} ; g_{1}, \ldots, g_{l-3} ; \sigma, \tau\right)$ by ${ }_{l+1} E_{l}^{(n)}(\sigma, \tau)$, so as to emphasize the dependence on the modular parameters. It is clear that ${ }_{l+1} E_{l}^{(n)}(\sigma, \tau)$ is meromorphic in $\sigma, \tau$ for $\sigma \in \mathbb{C}$ and $\tau$ in the upper half plane. The following theorem states (formally) that as function of these modular parameters the series ${ }_{l+1} E_{l}^{(n)}(\sigma, \tau)$ transforms as if it were a Jacobi function (i.e. a Jacobi form on $S L_{2}(\mathbb{Z})$ of weight and index zero [EZ]). For $n=1$ this result is due to Frenkel and Turaev [FT]. 
Theorem 4 (Modularity). (i). For generic values of the parameters $g_{0}, \ldots, g_{l-2}$ and $g$ subject to the balancing and truncation conditions in (6.5), the multiple modular hypergeometric series ${ }_{l+1} E_{l}^{(n)}(6.3)$ enjoys the following invariance with respect to the natural action of the group $S L_{2}(\mathbb{Z})$ on the modular parameters $(\sigma, \tau):$

$$
{ }_{l+1} E_{l}^{(n)}\left(\frac{\sigma}{c \tau+d}, \frac{a \tau+b}{c \tau+d}\right)={ }_{l+1} E_{l}^{(n)}(\sigma, \tau),
$$

where $a, b, c, d \in \mathbb{Z}$ such that $a d-b c=1$.

(ii). If, in addition to the conditions under (i), the parameters $g_{0}, \ldots, g_{r-2}$ and $g$ are also integer-valued, then ${ }_{l+1} E_{l}^{(n)}(\sigma, \tau)$ is elliptic in $\sigma$ :

$$
{ }_{l+1} E_{l}^{(n)}(\sigma+k+m \tau, \tau)={ }_{l+1} E_{l}^{(n)}(\sigma, \tau) \quad(k, m \in \mathbb{Z}) .
$$

Proof. The Jacobi $\theta_{1}$-function satisfies the following modular and quasiperiodicity relations $[\mathrm{WW}]$

$$
\left\{\begin{array} { l } 
{ \theta _ { 1 } ( x | \tau + 1 ) = e ^ { \pi i / 4 } \theta _ { 1 } ( x | \tau ) , } \\
{ \theta _ { 1 } ( \frac { x } { \tau } | - \frac { 1 } { \tau } ) = - i \sqrt { - i \tau } e ^ { \pi i x ^ { 2 } / \tau } \theta _ { 1 } ( x | \tau ) , }
\end{array} \quad \left\{\begin{array}{l}
\theta_{1}(x+1 \mid \tau)=-\theta_{1}(x \mid \tau) \\
\theta_{1}(x+\tau \mid \tau)=-e^{-\pi i \tau-2 \pi i x} \theta_{1}(x \mid \tau)
\end{array}\right.\right.
$$

These relations induce corresponding modular symmetries for the elliptic numbers $[x ; \sigma, \tau]$ :

$$
[x ; \sigma, \tau+1]=[x ; \sigma, \tau], \quad[x ; \sigma / \tau,-1 / \tau]=[x ; \sigma, \tau] e^{\pi i\left(x^{2}-1\right) \sigma / \tau},
$$

and

$$
\begin{aligned}
& {[x ; \sigma+1, \tau]=(-1)^{x-1}[x ; \sigma, \tau],} \\
& {[x ; \sigma+\tau, \tau]=(-1)^{x-1}[x ; \sigma, \tau] e^{\pi i\left(1-x^{2}\right)(\tau+2 \sigma)},}
\end{aligned}
$$

where in the second pair of identities it is assumed that $x$ is an integer. Since the action of $S L_{2}(\mathbb{Z})$ on the modular parameters is generated by the operations $(\sigma, \tau) \rightarrow(\sigma, \tau+1)$ and $(\sigma, \tau) \rightarrow(\sigma / \tau,-1 / \tau)$, it is clear from (6.9a) that for proving part (i) of the theorem it suffices to demonstrate that the sums of the squares of the arguments of the elliptic numbers in the numerator and denominator of the term ${ }_{l+1} \nu_{l}^{(n)}(\lambda ; \sigma, \tau)(6.4)$ are equal. A straightforward computation entails for the difference of these two sums of squares:

$$
\sum_{j=1}^{n} 2 \lambda_{j}\left(\lambda_{j}+2 \rho_{j}\right)\left((2 n-2) g+\sum_{r=0}^{l-2} g_{r}-(l-5) / 2\right),
$$

which is equal to zero in view of the balancing condition in (6.5). Similarly, it follows from $(6.9 \mathrm{~b})$ that to prove part (ii) we just need to show that in addition the difference between the sums of the arguments of the elliptic numbers in numerator and denominator is even when the parameters are integer-valued. 
We get for this difference

$$
\sum_{j=1}^{n} 2 \lambda_{j}\left((2 n-2) g+\sum_{r=0}^{l-2} g_{r}-(l-3) / 2\right),
$$

which is equal to $-\sum_{j=1}^{n} 2 \lambda_{j}=0 \bmod 2$ by the balancing condition in (6.5). This proves the stated properties (i) and (ii) for the terms of ${ }_{l+1} E_{l}^{(n)}(\sigma, \tau)(6.3)$ (and hence for the series itself).

At this point the summation of Corollary 2, and thus that of (6.7), hinges (for $n>1$ ) on the elliptic Macdonald-Morris conjecture of Section 3. We will now combine the fact that we have a complete proof of the sum for $p=0$ (cf. Theorem 3) with Theorem 4 and results from the theory of modular forms, to deduce that the multiple modular hypergeometric summation formula (6.7) holds at least up to order $\sigma^{10}$.

Theorem 5 (Asymptotics for $\sigma \rightarrow 0$ ). Let $g_{0}, \ldots, g_{5}$ and $g$ be generic parameters subject to the balancing and truncation conditions in (6.5) (with $l=7$ ). Then the Taylor expansions in $\sigma$ of both sides of the multidimensional modular hypergeometric summation formula (6.7), around the point $\sigma=0$, coincide (at least) up to the terms of order $\sigma^{10}$.

Proof. Let us temporarily assume that the parameters $g_{0}, \ldots, g_{5}$ and $g$ (subject to the balancing and truncation conditions) are real and generic in the sense that none of the arguments of the elliptic numbers arising in the summation formula (6.7) are equal to zero. Since the zeros of the elliptic numbers $[x ; \sigma, \tau]$ are located at $x=0 \bmod \frac{1}{\sigma} \mathbb{Z}+\frac{\tau}{\sigma} \mathbb{Z}$, this implies that for $\sigma$ real and sufficiently small both sides of the summation formula are holomorphic in $\tau$ in the upper half plane $\operatorname{Im}(\tau)>0$. Now, according to Theorem 4, the 1.h.s. of $(6.7)$ is invariant under the transformations $(\sigma, \tau) \rightarrow(\sigma, \tau+1)$ and $(\sigma, \tau) \rightarrow(\sigma / \tau,-1 / \tau)$. Just as in the proof of that theorem, it is not difficult to check - employing again the symmetries (6.9a) for the elliptic numbers - that the r.h.s. is invariant with respect to these transformations too. (Indeed, the difference between the sums of the squares of the arguments of the elliptic numbers in the numerator and denominator is given by $2 N\left(N+1+g_{0}-g_{1}-g_{2}-g_{3}\right) \sum_{j=1}^{n}(n+1-2 j) g=0$.) This modular invariance implies - together with the trivial observation that our expressions are even in $\sigma$ (because $[x ; \sigma, \tau]=[x ;-\sigma, \tau]$ ) - that the difference between the l.h.s. and r.h.s. of (6.7) has a Taylor expansion around $\sigma=0$ of the form

$$
\sum_{m \geq 0} c_{m}(\tau) \sigma^{2 m}
$$

where $c_{m}(\tau)$ is a modular form of weight $2 m$ on $S L_{2}(\mathbb{Z})$ (i.e., $c_{m}(\tau)$ is holomorphic on the upper half plane and $c_{m}\left(\frac{a \tau+b}{c \tau+d}\right)=(c \tau+d)^{2 m} c_{m}(\tau)$ for $a, b, c, d \in \mathbb{Z}$ with $a d-b c=1)$. Furthermore, since in the $\operatorname{limit} \operatorname{Im}(\tau) \rightarrow+\infty$ both sides of (6.7) are equal in view of Theorem 3 , we conclude that $\lim _{\tau \rightarrow+i \infty} c_{m}(\tau)=0$. In 
other words, $c_{m}(\tau)$ is a cusp form. Since the first nontrivial cusp form is a modular form of weight 12 [Se], it follows that the first nonzero term in the above Taylor expansion cannot appear before degree 12. The extension to generic complex parameters $g_{0}, \ldots, g_{5}$ and $g$ subject to the balancing and truncation conditions follows by analyticity.

It is clear from the symmetry property $(6.9 \mathrm{~b})$ of the elliptic numbers that for integer-valued parameters $g, g_{r}$ (subject to the balancing and truncation conditions in (6.5)) the r.h.s. of the summation formula (6.7) is elliptic in $\sigma$ too. Indeed, the difference between the sums of the arguments of the elliptic numbers in numerator and denominator equals $2 N \sum_{j=1}^{n}(n+1-2 j) g=0$. In other words, the summation conjecture may be regarded as a conjecture for an identity between elliptic functions of $\sigma$.

Theorems 3 and 5 (together with Frenkel-Turaev's proof for $n=1[\mathrm{FT}]$ and computer experiments for $n>1$ mentioned in [Wa]) provide additional evidence that Warnaar's multidimensional modular hypergeometric summation conjecture (6.7) indeed holds true. Furthermore, since we obtained this summation conjecture as a consequence of our elliptic Macdonald-Morris conjecture, it also provides further indication for the correctness of the latter conjecture.

Remark 5. In view of the proof of Theorem 5, we can also conclude that the terms of order $\sigma^{14}$ on both sides of (6.7) coincide. This is because there are no nontrivial cusp forms of weight 14 [Se]. It thus follows from our considerations that between the first 16 terms of the Tayor expansion in $\sigma$, ranging from degree 0 up to 15, a difference between the l.h.s and r.h.s. of (6.7) may appear at most in the terms of degree 12 .

Remark 6 . For $\sigma \rightarrow 0$ we have that $[x ; \sigma, \tau] \rightarrow x$. Hence, the equality of the constant terms of the Taylor series in $\sigma$ on both sides of (6.7) (following from Theorem 5) amounts to a multiple hypergeometric summation formula with all elliptic factorials replaced by ordinary shifted factorials $[x]_{m}=x(x+1) \cdots(x+$ $m-1)$. This formula is a multidimensional generalization of the classical 2balanced very-well-poised terminating ${ }_{7} F_{6}$ sum due to Dougall [GR]. It is a generalization to the ${ }_{7} F_{6}$ level of the multiple terminating ${ }_{5} F_{4}$ Dougall sum of [D, Theorem 3].

\section{Appendix A. Proof of the residue formula}

In this appendix we prove the residue formula of Theorem 1. The proof hinges on a residue formula for multivariate Askey-Wilson-type integrals due to Stokman $[\mathrm{St}]$. Let

$$
\text { (A.1) } \begin{aligned}
\bar{\Delta}_{n}(\mathbf{z} ; q)= & \frac{1}{(2 \pi i)^{n}} \prod_{1 \leq j<k \leq n} \frac{\left(z_{j} z_{k}, z_{j} z_{k}^{-1}, z_{j}^{-1} z_{k}, z_{j}^{-1} z_{k}^{-1} ; q\right)_{\infty}}{\left(t z_{j} z_{k}, t z_{j} z_{k}^{-1}, t z_{j}^{-1} z_{k}, t z_{j}^{-1} z_{k}^{-1} ; q\right)_{\infty}} \\
& \times \prod_{1 \leq j \leq n} \frac{\left(z_{j}^{2}, z_{j}^{-2} ; q\right)_{\infty}}{\left(t_{0} z_{j}, t_{0} z_{j}^{-1} ; q\right)_{\infty}},
\end{aligned}
$$


with $0<q, t<1$ and $t_{0} \in \mathbb{C} \backslash[1,+\infty[$. In [St] Stokman proved the following residue formula:

$$
\begin{aligned}
& \int_{C^{n}} \bar{\Delta}_{n}(\mathbf{z} ; q) f(\mathbf{z}) \frac{d z_{1}}{z_{1}} \cdots \frac{d z_{n}}{z_{n}}= \\
& \sum_{m=0}^{n} 2^{m} m !\left(\begin{array}{c}
n \\
m
\end{array}\right) \sum_{\substack{0 \leq \lambda_{1} \leq \cdots \leq \lambda_{m} \\
\left|\tau_{m} q^{\lambda} m\right|>1}} \int_{T^{n-m}} \bar{\mu}_{m}(\lambda, \mathbf{z} ; q) f_{m}(\lambda, z) \frac{d z_{1}}{z_{1}} \cdots \frac{d z_{n-m}}{z_{n-m}},
\end{aligned}
$$

where $\tau_{j}=t_{0} t^{j-1}$

$$
\begin{aligned}
\bar{\mu}_{m}(\lambda, \mathbf{z} ; q)= & \bar{\kappa}_{m} \bar{\nu}_{m}(\lambda ; q) \bar{\delta}_{m, n-m}(\lambda, \mathbf{z}) \bar{\Delta}_{n-m}(\mathbf{z} ; q), \\
f_{m}(\lambda, \mathbf{z})= & f\left(\tau_{1} q^{\lambda_{1}}, \ldots, \tau_{m} q^{\lambda_{m}}, z_{1}, \ldots, z_{n-m}\right), \\
\bar{\kappa}_{m}= & \prod_{1 \leq j<k \leq m} \frac{\left(\tau_{k} \tau_{j}^{-1}, \tau_{k}^{-1} \tau_{j}^{-1} ; q\right)_{\infty}}{\left(t \tau_{k} \tau_{j}^{-1}, t \tau_{k}^{-1} \tau_{j}^{-1} ; q\right)_{\infty}} \prod_{1 \leq j \leq m} \frac{\left(\tau_{j}^{-2} ; q\right)_{\infty}}{(q ; q)_{\infty}}, \\
\bar{\nu}_{m}(\lambda ; q)= & q^{-\sum_{j=1}^{m} \lambda_{j}\left(3 \lambda_{j}+1\right) / 2}\left(-t_{0}^{-4}\right)^{\sum_{j=1}^{m} \lambda_{j}} t^{-5 \sum_{j=1}^{m}(j-1) \lambda_{j}} \\
& \times \prod_{1 \leq j<k \leq m}\left(\frac{1-\tau_{k} \tau_{j} q^{\lambda_{k}+\lambda_{j}}}{1-\tau_{k} \tau_{j}} \frac{1-\tau_{k} \tau_{j}^{-1} q^{\lambda_{k}-\lambda_{j}}}{1-\tau_{k} \tau_{j}^{-1}}\right) \\
& \times \frac{\left(t \tau_{k} \tau_{j} ; q\right)_{\lambda_{k}+\lambda_{j}}}{\left(q t^{-1} \tau_{k} \tau_{j} ; q\right)_{\lambda_{k}+\lambda_{j}}} \frac{\left(t \tau_{k} \tau_{j}^{-1} ; q\right)_{\lambda_{k}-\lambda_{j}}}{\left(q t^{-1} \tau_{k} \tau_{j}^{-1} ; q\right)_{\lambda_{k}-\lambda_{j}}} \\
& \times \prod_{1 \leq j \leq m}\left(\frac{1-\tau_{j}^{2} q^{2 \lambda_{j}}}{1-\tau_{j}^{2}}\right) \frac{\left(t_{0} \tau_{j} ; q\right)_{\lambda_{j}}}{\left(q t_{0}^{-1} \tau_{j} ; q\right)_{\lambda_{j}}},
\end{aligned}
$$

and

$$
\bar{\delta}_{m, n-m}(\lambda, \mathbf{z})=\prod_{\substack{1 \leq j \leq m \\ 1 \leq k \leq n-m}} \frac{\left(\tau_{j} q^{\lambda_{j}} z_{k}, \tau_{j} q^{\lambda_{j}} z_{k}^{-1}, \tau_{j}^{-1} q^{-\lambda_{j}} z_{k}, \tau_{j}^{-1} q^{-\lambda_{j}} z_{k}^{-1} ; q\right)_{\infty}}{\left(t \tau_{j} q^{\lambda_{j}} z_{k}, t \tau_{j} q^{\lambda_{j}} z_{k}^{-1}, t \tau_{j}^{-1} q^{-\lambda_{j}} z_{k}, t \tau_{j}^{-1} q^{-\lambda_{j}} z_{k}^{-1} ; q\right)_{\infty}}
$$

Here $T$ is the positively oriented unit circle and $C \subset \mathbb{C}$ is a positively oriented (smooth) Jordan curve around zero such that $(i)$ every half-line parting from zero intersects $C$ once, (ii) $C^{-1}:=\left\{z \in \mathbb{C} \mid z^{-1} \in C\right\}=C$, and (iii) $C$ separates the poles at $\left\{t_{0} q^{l}\right\}_{l \in \mathbb{N}}$ (in the interior) from the poles at $\left\{t_{0}^{-1} q^{-l}\right\}_{l \in \mathbb{N}}$ (in the exterior). Furthermore, $f(\mathbf{z})$ denotes an arbitrary (complex) symmetric and $z_{j} \rightarrow z_{j}^{-1}$ reflection invariant function in $z_{1}, \ldots, z_{n}$ that is assumed to be holomorphic for $z_{j}$ in the closure of the symmetric difference of $\operatorname{Int}(C)$ and $\operatorname{Int}(T)$.

In order to demonstrate that the residue formula of Theorem 1 follows from (A.2), we choose the parameters and integration contour $C$ in correspondence 
with the conditions of the theorem. Clearly the $S_{n} \ltimes \mathbb{Z}_{2}^{n}$ symmetric function

$$
f(\mathbf{z})=\frac{\Delta_{n}(\mathbf{z} ; p, q)}{\bar{\Delta}_{n}(\mathbf{z} ; q)}
$$

is then holomorphic in $z_{j}$ inside the closure of the symmetric difference of $\operatorname{Int}(C)$ and $\operatorname{Int}(T)$. (Here $\Delta_{n}(\mathbf{z} ; p, q)$ is given by (3.1).) Indeed, the poles of $\Delta_{n}(\mathbf{z} ; p, q)$ at $z_{j}^{ \pm 1}=t_{0} q^{l}$ with $\left|t_{0} q^{l}\right|>1$ are compensated by corresponding poles of $\bar{\Delta}_{n}(\mathbf{z} ; q)$. Substituting $f(\mathbf{z})$ (A.3) into (A.2) now entails the formula of Theorem 1 in view of the iterated limit

$$
\begin{aligned}
f_{m}(\lambda, \mathbf{z}) & =\lim _{y_{m} \rightarrow \tau_{m} q^{\lambda_{m}}} \cdots \lim _{y_{1} \rightarrow \tau_{1} q^{\lambda_{1}}} f\left(y_{1}, \ldots, y_{m}, z_{1}, \ldots, z_{n-m}\right) \\
& =\frac{\mu_{m}(\lambda, \mathbf{z} ; p, q)}{\bar{\mu}_{m}(\lambda, \mathbf{z} ; q)}
\end{aligned}
$$

(where the function $\mu_{m}(\lambda, \mathbf{z} ; p, q)$ is defined in the theorem). This limit follows inductively from the somewhat tedious but straightforward limiting relation

$$
\lim _{z_{n-m+1} \rightarrow \tau_{m} q^{\lambda_{m}}} \frac{\mu_{m-1}(\lambda, \mathbf{z} ; p, q)}{\bar{\mu}_{m-1}(\lambda, \mathbf{z} ; q)}=\frac{\mu_{m}(\lambda, \mathbf{z} ; p, q)}{\bar{\mu}_{m}(\lambda, \mathbf{z} ; q)}
$$

(using also the permutation-invariance).

\section{References}

[AW] R. Askey and J. Wilson, Some basic hypergeometric orthogonal polynomials that generalize Jacobi polynomials, Mem. Amer. Math. Soc. 54 (1985).

[A] K. Aomoto, On elliptic product formulas for Jackson integrals associated with reduced root systems, J. Algebraic Combin. 8 (1998), 115-126.

$[\mathrm{BF}]$ T. H. Baker and P. J. Forrester, Transformation formulas for multivariable basic hypergeometric series, Methods Appl. Anal. 6 (1999), 147-164.

[BS] E. P. van den Ban and H. Schlichtkrull, A residue calculus for root systems, Compositio Math. 123 (2000), 27-72.

[D] J. F. van Diejen, On certain multiple Bailey, Rogers, and Dougall type summation formulas, Publ. Res. Inst. Math. Sci. 33 (1997), 483-508.

[EZ] M. Eichler and D. Zagier, The Theory of Jacobi Forms, Progress in Mathematics 55, Birkhäuser, Boston, 1985.

[FV] G. Felder and A. Varchenko, The elliptic gamma function and $S L(3, \mathbb{Z}) \ltimes \mathbb{Z}^{3}$, Adv. Math., to appear.

[FT] I. B. Frenkel and V. G. Turaev, Elliptic solutions of the Yang-Baxter equation and modular hypergeometric functions, The Arnold-Gelfand Mathematical Seminars (Eds. V. I. Arnold, I. M. Gelfand, V. S. Retakh, and M. Smirnov), Birkhäuser, Boston, 1997, pp. 171-204.

[G1] R. A. Gustafson, A generalization of Selberg's beta integral, Bull. Amer. Math. Soc. (N.S.) 22 (1990), 97-105.

[G2] S Some q-beta Integrals on $S U(n)$ and $S p(n)$ that generalize the Askey-Wilson and Nassrallah-Rahman integrals, SIAM J. Math. Anal. 25 (1994), 441-449.

[GR] G. Gasper and M. Rahman, Basic Hypergeometric Series, Encyclopedia of Mathematics and its Applications 35, Cambridge University Press, Cambridge, 1990.

[HO] G. J. Heckman and E. M. Opdam, Yang's system of particles and Hecke algebras, Ann. of Math. 145 (1997), 139-173, erratum 146 (1997), 749-750. 
[I] M. Ito, On a theta product formula for Jackson integrals associated with root systems of rank two, J. Math. Anal. Appl. 216 (1997), 122-163.

[K] K. W. J. Kadell, A proof of the q-Macdonald-Morris conjecture for $B C_{n}$, Mem. Amer. Math. Soc. 108 (1994).

[Ma1] I. G. Macdonald, A formal identity for affine root systems, Preprint, 1996.

[Ma2] _ Constant term identities, orthogonal polynomials, and affine Hecke algebras, Doc. Math. (1998), DMV Extra Volume ICM I, 303-317.

[M1] S. C. Milne, Multiple q-series and $U(n)$ generalizations of Ramanujan's ${ }_{1} \Psi_{1}$ sum. Ramanujan Revisited, (Eds. G.E. Andrews, R.A. Askey, B.C. Berndt, K.G. Ramanathan, and R.A. Rankin), Academic Press, Boston, 1988, pp. 473-524.

[M2] The multidimensional ${ }_{1} \Psi_{1}$ sum and Macdonald identities for $A_{l}^{(1)}$. Theta Functions Bowdoin 1987, (Eds. L. Ehrenpreis and R.C. Gunning), Proc. Sympos. Pure Math. 49 (part 2), Amer. Math. Soc., Providence, R.I., 1989, pp. 323-359.

[ML] S. C. Milne and G. M. Lilly, Consequences of the $A_{l}$ and $C_{l}$ Bailey transform and Bailey lemma, Discrete Math. 139 (1995), 319-346.

[NR] B. Nassrallah and M. Rahman, Projection formulas, a reproducing kernel and a generating function for q-Wilson polynomials, SIAM J. Math. Anal. 16 (1985), 186-197.

[R1] M. Rahman, An integral representation of a ${ }_{10} \phi_{9}$ and continuous bi-orthogonal ${ }_{10} \phi_{9}$ rational functions, Canad. J. Math. 38 (1986), 605-618.

[R2] - Biorthogonality of a system of rational functions with respect to a positive measure on [-1, 1], SIAM J. Math. Anal. 22 (1991), 1430-1441.

[Ru] S. N. M. Ruijsenaars, First order analytic difference equations and integrable quantum systems, J. Math. Phys. 38 (1997), 1069-1146.

[Sc] M. Schlosser, Summation theorems for multidimensional basic hypergeometric series by determinant evaluations, Discrete Math. 210 (2000), 151-169.

[Se] J.-P. Serre, A Course in Arithmetic, Springer Verlag, New York, 1973.

[S] V. P. Spiridonov, An elliptic beta integral, Proceedings of the Fifth International Conference on Difference Equations and Applications (Temuco, Chile, January 3-7, 2000), (Eds. S. Elaydi, J. Fenner-Lopez, and G. Ladas), Gordon and Breach, to appear.

[SZ] V. P. Spiridonov and A. S. Zhedanov, Spectral transformation chains and some new biorthogonal rational functions, Comm. Math. Phys. 210 (2000), 49-83.

[St] J. V. Stokman, On BC type basic hypergeometric orthogonal polynomials, Trans. Amer. Math. Soc. 352 (2000), 1527-1579.

[Wa] S. O. Warnaar, Summation and transformation formulas for elliptic hypergeometric series, Preprint, 2000.

[WW] E. T. Whittaker and G. N. Watson, A Course of Modern Analysis, Cambridge University Press, Cambridge, 1986.

[W1] J. A. Wilson, Hypergeometric Series, Recurrence Relations and Some New Orthogonal Functions, Ph. D. thesis, University of Wisconsin, Madison, WI, 1978.

[W2] _ Orthogonal functions from Gram determinants, SIAM J. Math. Anal. 22 (1991), 1147-1155.

Departamento de Matemáticas, Facultad de Ciencias, Universidad de Chile, Casilla 653, Santiago 1, Chile

E-mail address: vandiej@uchile.cl

Bogoliubov Laboratory of Theoretical Physics, Joint Institute for Nuclear ReSEARCh, Dubna, Moscow Region 141980, Russia

E-mail address: svp@thsun1.jinr.ru 